CRYSTALLOGRAPHIC COMMUNICATIONS

ISSN 2056-9890

Received 27 September 2021

Accepted 7 October 2021

Edited by W. T. A. Harrison, University of Aberdeen, Scotland

Keywords: crystal structure; polymorphism; cobalt(II)thiocyanate; hydrogen bonding.

CCDC references: 2114553; 2114552

Supporting information: this article has supporting information at journals.iucr.org/e

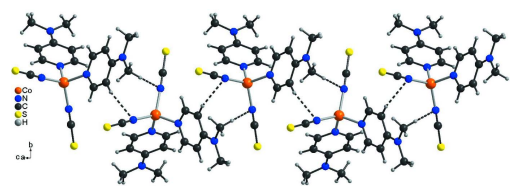

\section{Comparison of the crystal structures of the low- and high-temperature forms of bis[4-(dimethylamino)- pyridine]dithiocyanatocobalt(II)}

\author{
Christoph Krebs, ${ }^{*}$ Inke Jess and Christian Näther
}

Institute of Inorganic Chemistry, University of Kiel, Max-Eyth.-Str. 2, 24118 Kiel, Germany. *Correspondence e-mail: ckrebs@ac.uni-kiel.de

Single crystals of the high-temperature form I of $\left[\mathrm{Co}(\mathrm{NCS})_{2}(\mathrm{DMAP})_{2}\right]$ (DMAP = 4-dimethylaminopyridine, $\mathrm{C}_{7} \mathrm{H}_{10} \mathrm{~N}_{2}$ ) were obtained accidentally by the reaction of $\mathrm{Co}(\mathrm{NCS})_{2}$ with DMAP at slightly elevated temperatures under kinetic control. This modification crystallizes in the monoclinic space group $P 2_{1} /$ $m$ and is isotypic with the corresponding $\mathrm{Zn}$ compound. The asymmetric unit consists of one crystallographically independent Co cation and two crystallographically independent thiocyanate anions that are located on a crystallographic mirror plane and one DMAP ligand (general position). In its crystal structure the discrete complexes are linked by $\mathrm{C}-\mathrm{H} \cdots \mathrm{S}$ hydrogen bonds into a three-dimensional network. For comparison, the crystal structure of the known low-temperature form II, which is already thermodynamically stable at room temperature, was redetermined at the same temperature. In this polymorph the complexes are connected by $\mathrm{C}-\mathrm{H} \cdots \mathrm{S}$ and $\mathrm{C}-\mathrm{H} \cdots \mathrm{N}$ hydrogen bonds into a three-dimensional network. At $100 \mathrm{~K}$ the density of the high-temperature form I $\left(\rho=1.462 \mathrm{~g} \mathrm{~cm}^{-3}\right)$ is higher than that of the low-temperature form II $(\rho=$ $1.457 \mathrm{~g} \mathrm{~cm}^{-3}$ ), which is in contrast to the values determined by XRPD at room temperature. Therefore, these two forms represent an exception to the Kitaigorodskii density rule, for which extensive intermolecular hydrogen bonding in form II might be responsible.

\section{Chemical context}

Polymorphism and isomerism is a widespread phenomenon in coordination chemistry (Braga \& Grepioni, 2000; Moulton \& Zaworotko, 2001; Batten et al., 1998; Zhang et al., 2009). On one hand, these phenomena are a disadvantage for rational crystal design, but on the other hand they are of advantage for studying structure-property relationships (Braga et al., 2001; Tao et al., 2012; Ossinger et al., 2020; Sheu et al., 2009). Because in such a case the composition of the different forms is identical, all changes in the physical properties can be directly correlated with the structural changes. One class of compounds in which polymorphism and especially isomerism is observed are coordination compounds based on transitionmetal thiocyanates, because this anionic ligand shows several different coordination modes leading to a large structural variability (Böhme et al., 2020; Jochim et al., 2020; Mautner et al., 2018; Neumann et al., 2020a; Wellm et al., 2020a; Werner et al., 2015; Buckingham, 1994; Barnett et al., 2002).

In this context, we have recently reported the crystal structure of form II of $\mathrm{Co}(\mathrm{NCS})_{2}(\mathrm{DMAP})_{2}(\mathrm{DMAP}=4$-dimethylaminopyridine, $\mathrm{C}_{7} \mathrm{H}_{10} \mathrm{~N}_{2}$ ), which crystallizes as discrete complexes in which the cobalt cations are tetrahedrally coor- 
dinated. This modification can directly be obtained from the reaction of $\mathrm{Co}(\mathrm{NCS})_{2}$ and 4-dimethylaminopyridine in aqueous solution or by thermal decomposition of $\mathrm{Co}(\mathrm{NCS})_{2}(\mathrm{DMAP})_{2}\left(\mathrm{H}_{2} \mathrm{O}\right)_{2}$-dihydrate (Neumann et al., 2018a). In contrast, if the methanol complex $\mathrm{Co}(\mathrm{NCS})_{2}(\mathrm{DMAP})_{2}(\mathrm{MeOH})_{2}$ is thermally decomposed, a new polymorphic modification of $\mathrm{Co}(\mathrm{NCS})_{2}(\mathrm{DMAP})_{2}$ (form I) is obtained. Because we were not able to prepare single crystals of this form, the corresponding $\mathrm{Zn}$ complex was prepared and XRPD indicates that it is isotypic to form I of the Co compound (Neumann et al., 2018b). Solvent-mediated conversion experiments reveal that form II is the thermodynamically stable form at room temperature and transforms into form I upon heating. Both forms are related by enantiotropism and the thermodynamic transition temperature was determined to be above $135^{\circ} \mathrm{C}$. The metastability of form I at room temperature might be the reason why no single crystals were obtained. It is noted that in contrast to the Co modification I, the corresponding $\mathrm{Zn}$ form is already thermodynamically stable at room temperature, which might be the reason that single crystals of this form can easily be prepared from solution (Neumann et al., 2020a,b).

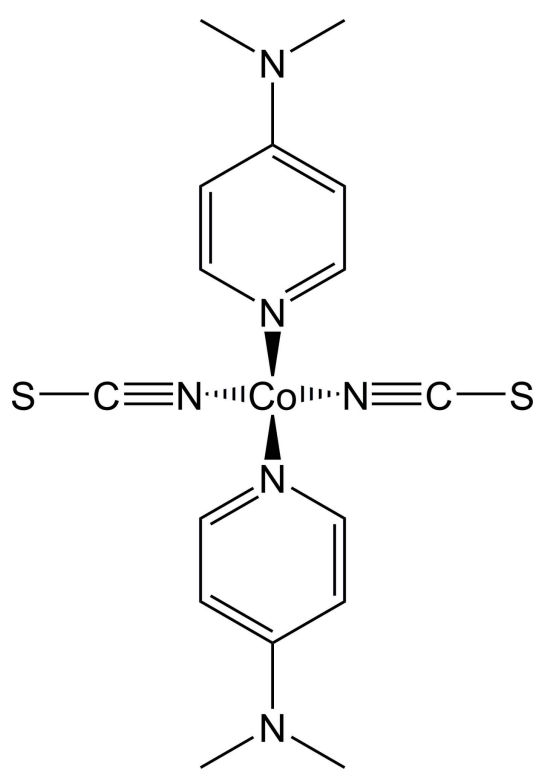

Later on, we investigated whether the physical properties of thiocyanate coordination compounds can be influenced by mixed crystal formation and we found out that, for example, the critical temperature in layered thiocyanate networks can be tuned by preparing mixed crystals with $\mathrm{Co}(\mathrm{NCS})_{2}$ and $\mathrm{Ni}(\mathrm{NCS})_{2}$ where a linear increase of $T_{\mathrm{c}}$ with increasing Co content was observed (Neumann et al., 2018b; Wellm et al., $2018,2020 b$ ). In the course of our systematic work, we are currently investigating whether mixed crystals of $\mathrm{Ni}(\mathrm{NCS})_{2}(\mathrm{DMAP})_{2}$ and $\mathrm{Co}(\mathrm{NCS})_{2}(\mathrm{DMAP})_{2}$ can be prepared. As already noted, the Co compound forms discrete complexes whereas the Ni compound shows a chain structure (Jochim et al., 2018). Preliminary XRPD investigations indicate that, in those cases where more than $50 \% \mathrm{Co}(\mathrm{NCS})_{2}$ is used in the synthesis, a very small amount of form I of $\mathrm{Co}(\mathrm{NCS})_{2}(\mathrm{DMAP})_{2}$ is formed as a side phase. This is in agreement with crystallization experiments to obtain single crystals where $\mathrm{Co}(\mathrm{NCS})_{2}$ and $\mathrm{Ni}(\mathrm{NCS})_{2}$ were used in a 90:10 ratio, because block-shaped and needle-like crystal are visible. Both of them were identified by single crystal X-ray diffraction, which proves that the block-like crystals correspond to the unit cell of the $\mathrm{Ni}$ compound, whereas the needle-like crystals correspond to the metastable form $\mathrm{I}$ of $\mathrm{Co}(\mathrm{NCS})_{2}(\mathrm{DMAP})_{2}$, which was obviously obtained accidentally under kinetic control. To exclude the possibility that mixed crystals of form I have formed, the crystallization reaction was repeated with only $\mathrm{Co}(\mathrm{NCS})_{2}$ and in this case the same crystalline phase was obtained. As mentioned above, its single-crystal structure is unknown and it is therefore presented here for the first time. For better comparison, we also present the structure of form II at $100 \mathrm{~K}$, because in our previous work it was measured at $170 \mathrm{~K}$ (Neumann et al., 2018b).

\section{Structural commentary}

Form I of $\mathrm{Co}(\mathrm{NCS})_{2}(\mathrm{DMAP})_{2}$ crystallizes in the monoclinic space group $P 2_{1} / m$ with $Z=2$ and the Co cation as well as the thiocyanate anions are located on a crystallographic mirror

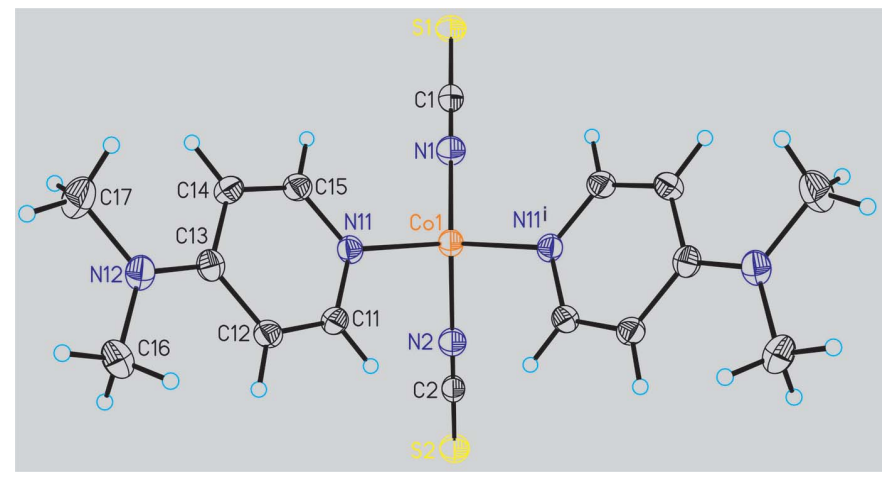

Figure 1

Crystal structure of form I with labeling and displacement ellipsoids drawn at the $50 \%$ probability level. Symmetry code: (i) $=x, 3 / 2-\mathrm{y}, z$.

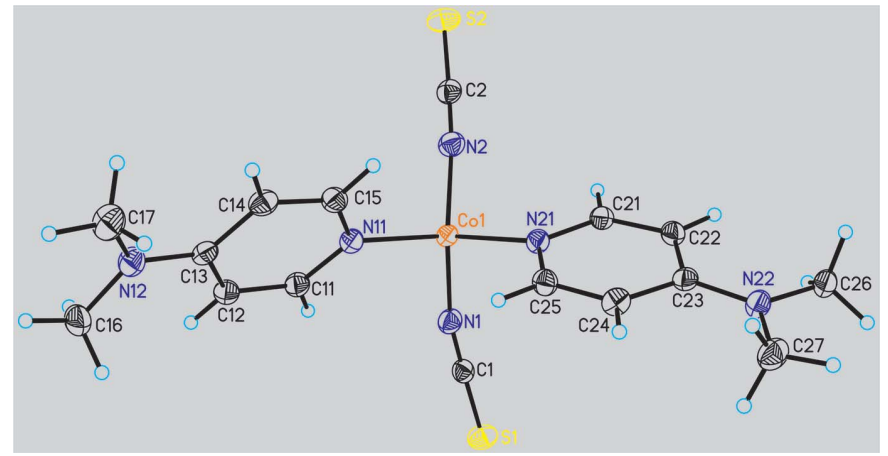

Figure 2

Crystal structure of form II with labeling and displacement ellipsoids drawn at the $50 \%$ probability level. 
Table 1

Selected geometric parameters $\left(\AA,^{\circ}\right)$ for form I.

\begin{tabular}{lrll}
\hline Co1-N1 & $1.9429(18)$ & Co1-N11 & $2.0148(12)$ \\
Co1-N2 & $1.9672(19)$ & & \\
& & & \\
N1-Co1-N2 & $118.16(8)$ & N11-Co1-N11 & $109.04(7)$ \\
N1-Co1-N11 & $111.03(4)$ & C1-N1-Co1 & $179.48(17)$ \\
N2-Co1-N11 & $103.47(4)$ & C2-N2-Co1 & $166.24(16)$ \\
\hline
\end{tabular}

Symmetry code: (i) $x,-y+\frac{3}{2}, z$.

Table 2

Selected geometric parameters $\left(\AA{ }^{\circ}\right)$ for form II.

\begin{tabular}{lrll}
\hline Co1-N1 & $1.9521(13)$ & Co1-N11 & $2.0057(12)$ \\
Co1-N2 & $1.9535(14)$ & Co1-N21 & $2.0013(12)$ \\
& & & \\
N1-Co1-N2 & $117.81(6)$ & N2-Co1-N21 & $106.71(5)$ \\
N1-Co1-N11 & $105.83(5)$ & N21-Co1-N11 & $112.36(5)$ \\
N1-Co1-N21 & $105.41(5)$ & C1-N1-Co1 & $168.56(12)$ \\
N2-Co1-N11 & $108.82(5)$ & $\mathrm{C} 2-\mathrm{N} 2-\mathrm{Co} 1$ & $175.34(13)$ \\
\hline
\end{tabular}

plane, whereas the known form II crystallizes in space group $P 2_{1} / c$ with $Z=4$ with all atoms in general positions. In both modifications, the $\mathrm{Co}^{\mathrm{II}}$ cations are fourfold coordinated by two terminal $\mathrm{N}$-bonded thiocyanate anions and two DMAP ligands within slightly distorted tetrahedral environments (Figs. 1 and 2 and Table 1). In form I, the two $\mathrm{Co}-\mathrm{N}$ bond lengths to the thiocyanate anions are slightly different, which is not the case in form II (Table 2). Usually this is reflected in the values of the $\mathrm{CN}$ stretching vibrations but this is not the case for form I, because two bands are expected but only one is visible in its IR spectrum (Neumann et al., 2018b). Moreover, the $\mathrm{Co}-\mathrm{N}$ bond lengths to the DMAP ligands are slightly longer in form I compared to form II (Table 2). From the $\mathrm{N}-\mathrm{Co}-\mathrm{N}$ bond angles, it is obvious that both tetrahedra are slightly distorted (Table 1 ). In both modifications, the $\mathrm{Co}-$ $\mathrm{N}-\mathrm{C}$ bond angle is close to linear. Finally, it is noted that the density of form I at $100 \mathrm{~K}$ of $1.462 \mathrm{~g} \mathrm{~cm}^{-3}$ is significantly greater than that of form II $\left(1.457 \mathrm{~g} \mathrm{~cm}^{-3}\right)$. This is surprising because form I was proven to be thermodynamically stable at a lower temperature and should have the higher density according to the density rule (Kitaigorodskii, 1961). This was determined from a Pawley fit of a powder pattern measured at room temperature (Neumann et al., 2018b) and therefore, the current findings are somehow in contradiction to the previous findings. Other exceptions to this rule are known if the crystal structure is dominated by intermolecular hydrogen bonding, as already discussed in the literature (Burger \& Ramberger, 1979).

\section{Supramolecular features}

In the crystal structure of form I, the discrete complexes are linked by $\mathrm{C}-\mathrm{H} \cdots \mathrm{S}$ hydrogen bonds between one of the DMAP methyl $\mathrm{H}$ atoms and the thiocyanate $\mathrm{S}$ atoms into layers that lie parallel to the $b c$ plane (Fig. 3). In this arrangement, each of the two $\mathrm{S}$ atoms acts as an acceptor for two hydrogen bonds to two symmetry-equivalent DMAP ligands (Fig. 3). The $\mathrm{C}-\mathrm{H} \cdots \mathrm{S}$ angles are close to $180^{\circ}$, indicating a relatively strong interaction (Table 3 ). These layers
Table 3

Hydrogen-bond geometry $\left(\AA,^{\circ}\right)$ for form I.

\begin{tabular}{lllll}
\hline$D-\mathrm{H} \cdots A$ & $D-\mathrm{H}$ & $\mathrm{H} \cdots A$ & $D \cdots A$ & $D-\mathrm{H} \cdots A$ \\
\hline $\mathrm{C} 16-\mathrm{H} 16 B \cdots \mathrm{S} 2^{\mathrm{ii}}$ & 0.98 & 2.91 & $3.8367(15)$ & 158 \\
$\mathrm{C} 17-\mathrm{H} 17 B \cdots \mathrm{S} 1^{\mathrm{iii}}$ & 0.98 & 2.94 & $3.7291(16)$ & 138 \\
$\mathrm{C} 17-\mathrm{H} 17 C \cdots \mathrm{S}^{\text {iv }}$ & 0.98 & 2.89 & $3.8018(18)$ & 155 \\
\hline
\end{tabular}

Symmetry codes: (ii) $-x+1,-y+1,-z+1$; $\quad$ (iii) $\quad-x+1,-y+1,-z$; (iv) $-x+2,-y+1,-z$.

Table 4

Hydrogen-bond geometry $\left(\AA,^{\circ}\right)$ for form II.

\begin{tabular}{lllll}
\hline$D-\mathrm{H} \cdots A$ & $D-\mathrm{H}$ & $\mathrm{H} \cdots A$ & $D \cdots A$ & $D-\mathrm{H} \cdots A$ \\
\hline $\mathrm{C} 11-\mathrm{H} 11 \cdots \mathrm{S} 1^{\mathrm{i}}$ & 0.95 & 2.87 & $3.7312(15)$ & 152 \\
$\mathrm{C} 16-\mathrm{H} 16 C \cdots \mathrm{S} 2^{\mathrm{ii}}$ & 0.98 & 3.01 & $3.9663(16)$ & 166 \\
$\mathrm{C} 21-\mathrm{H} 21 \cdots \mathrm{S} 1^{\text {iii }}$ & 0.95 & 2.92 & $3.7888(15)$ & 153 \\
$\mathrm{C} 22-\mathrm{H} 22 \cdots \mathrm{N} 1^{\text {iv }}$ & 0.95 & 2.64 & $3.5448(19)$ & 159 \\
$\mathrm{C} 26-\mathrm{H} 26 A \cdots \mathrm{N} 2^{\text {iv }}$ & 0.98 & 2.70 & $3.531(2)$ & 143 \\
$\mathrm{C} 27-\mathrm{H} 27 B \cdots \mathrm{S} 2^{\text {iv }}$ & 0.98 & 2.98 & $3.7568(17)$ & 137 \\
\hline
\end{tabular}

Symmetry codes: (i) $-x+2,-y+1,-z+2$; (ii) $-x+1,-y+1,-z+2$; $\quad$ (iii) $-x+2, y-\frac{1}{2},-z+\frac{3}{2}$; (iv) $x,-y+\frac{1}{2}, z-\frac{1}{2}$.

are further connected by weaker $\mathrm{C}-\mathrm{H}$ - . S contacts involving the thiocyanate $\mathrm{S}$ atom $\mathrm{S} 1$ and the methyl $\mathrm{H}$ atoms of the DMAP ligands (Fig. 4).

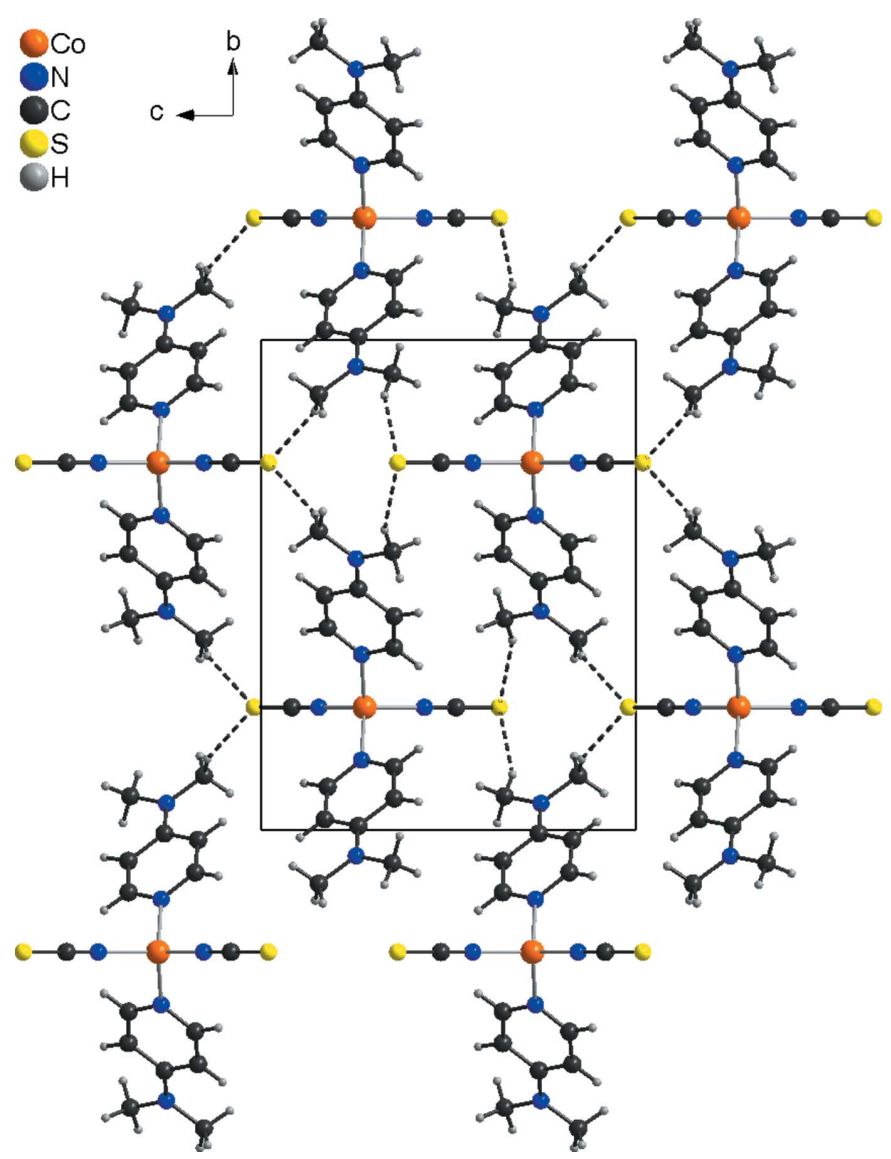

Figure 3

Crystal structure of form I with a view of a layer in the direction of the crystallographic $a$-axis. Intermolecular $\mathrm{C}-\mathrm{H} \cdots \mathrm{S}$ hydrogen bonding is shown as dashed lines. 


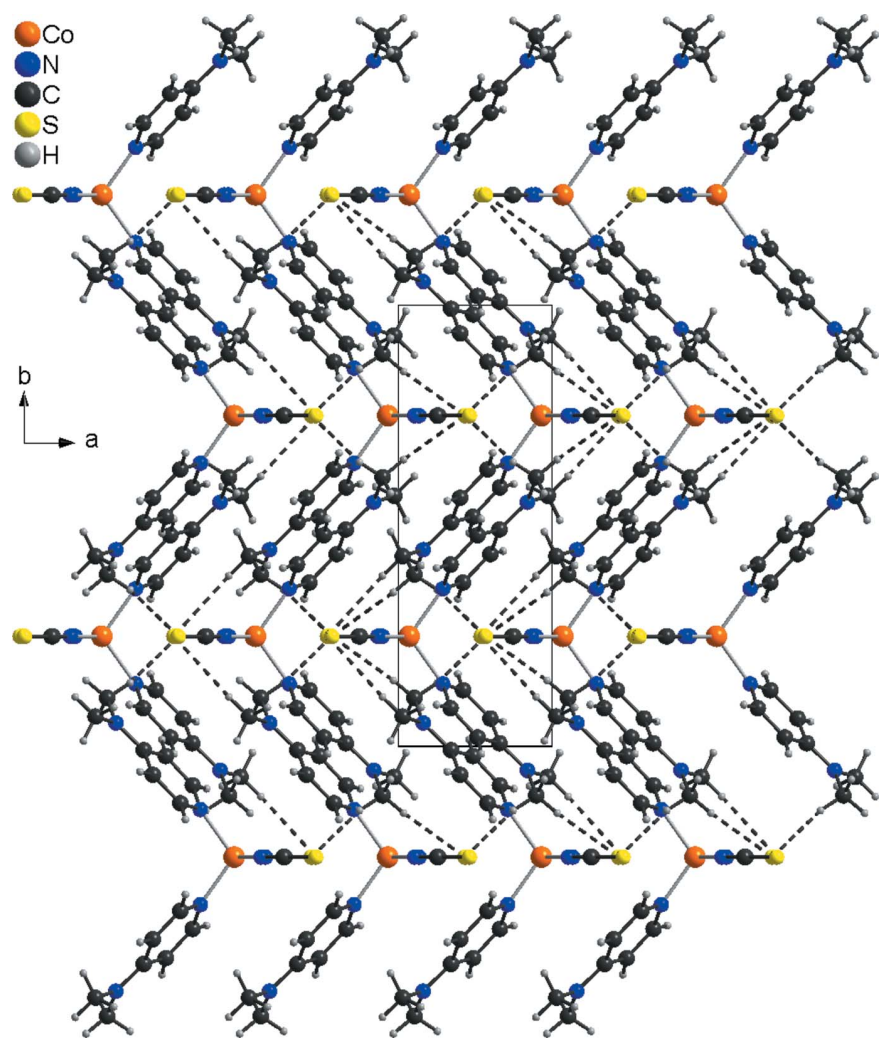

Figure 4

Crystal structure of form I viewed in the direction of the crystallographic $c$-axis. Intermolecular $\mathrm{C}-\mathrm{H} \cdots \mathrm{S}$ hydrogen bonding is shown as dashed lines.

In contrast to form I, both hydrogen bonds, $\mathrm{C}-\mathrm{H} \cdots \mathrm{S}$ and $\mathrm{C}-\mathrm{H} \cdots \mathrm{N}$, are present in form II. In this modification, the molecules are linked by pairs of $\mathrm{C}-\mathrm{H} \cdots \mathrm{N}$ hydrogen bonds between the thiocyanate $\mathrm{N}$ atoms and the $\mathrm{H}$ atoms of the DMAP ligands into chains that propagate along the crystallographic $c$-axis direction (Fig. 5). These chains are further linked into a complicated three-dimensional network by four different $\mathrm{C}-\mathrm{H}$...S hydrogen bonds between the hydrogen atoms of the DMAP ligands and the thiocyanate $\mathrm{S}$ atoms (Fig. 6 and Table 4). For three of these hydrogen bonds, the $\mathrm{C}-\mathrm{H} \cdots \mathrm{S}$ angle is close to linearity, which indicates that it is a relatively strong interaction. This extensive intermolecular hydrogen bonding might be responsible for the fact that the density of the low-temperature form II at $100 \mathrm{~K}$ is lower than

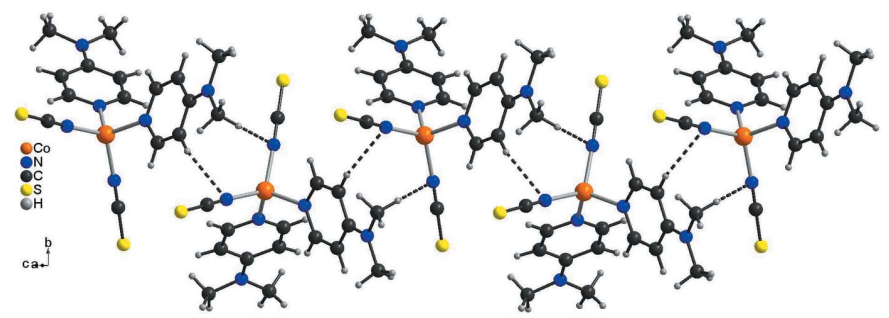

Figure 5

Crystal structure of form II with a view of a chain with intermolecular C$\mathrm{H} \cdots \mathrm{S}$ hydrogen bonding shown as dashed lines. that of the high-temperature form I, which is an exception to the density rule.

\section{Database survey}

As mentioned in the Chemical context section, the singlecrystal structure of form II and the thermodynamic relations between form I and form II have already been reported (Neumann et al., 2018b). Also related are the corresponding $\mathrm{Zn}(\mathrm{NCS})_{2}$ modifications, but in contrast to $\mathrm{Co}$, three different forms were observed with Zn (Neumann et al., 2018a,b).

However, compounds with DMAP and other transitionmetal thiocyanates also exist. This includes the compound $\mathrm{Zn}(\mathrm{NCS})_{2}(\mathrm{DMAP})_{2} \cdot$ chlorobenzene (Cambridge Structural Database refcode: QIPXES; Secondo et al., 2000), where the metal center is tetrahedrally coordinated. In addition, some octahedral complexes are known in the literature. $\mathrm{Cu}(\mathrm{NCS})_{2}(\mathrm{DMAP})_{2}$ (dimethylformamide) ${ }_{2}$ (HIVZAO; Chen et al., 2007), $\mathrm{Mn}(\mathrm{NCS})_{2}(\mathrm{DMAP})_{2}\left(\mathrm{CH}_{3} \mathrm{OH}\right)_{2} \quad$ (NUKCON; Suckert et al., 2015) and $\mathrm{Cd}(\mathrm{NCS})_{2}(\mathrm{DMAP})_{2}(\mathrm{DMSO})_{2}$ (QIPXOC; Secondo et al., 2000) all consist of a metal center with two thiocyanate anions, two DMAP co-ligands and two additional identical co-ligands each.

In $\left[\mathrm{Cd}(\mathrm{NCS})_{2}(\mathrm{DMAP})_{2}\right]_{n}$ (QIPXIW; Secondo et al., 2000) and $\left[\mathrm{Ni}(\mathrm{NCS})_{2}(\mathrm{DMAP})_{2}\right]_{n}$ (GIQQOP; Jochim et al., 2018), two non-isotypical linear chains are reported, in which the cations have an all-trans $M \mathrm{~N}_{4} \mathrm{~S}_{2}$ octahedral coordination of two N-bonded and two S-bonded bridging thiocyanate anions and two DMAP co-ligands.

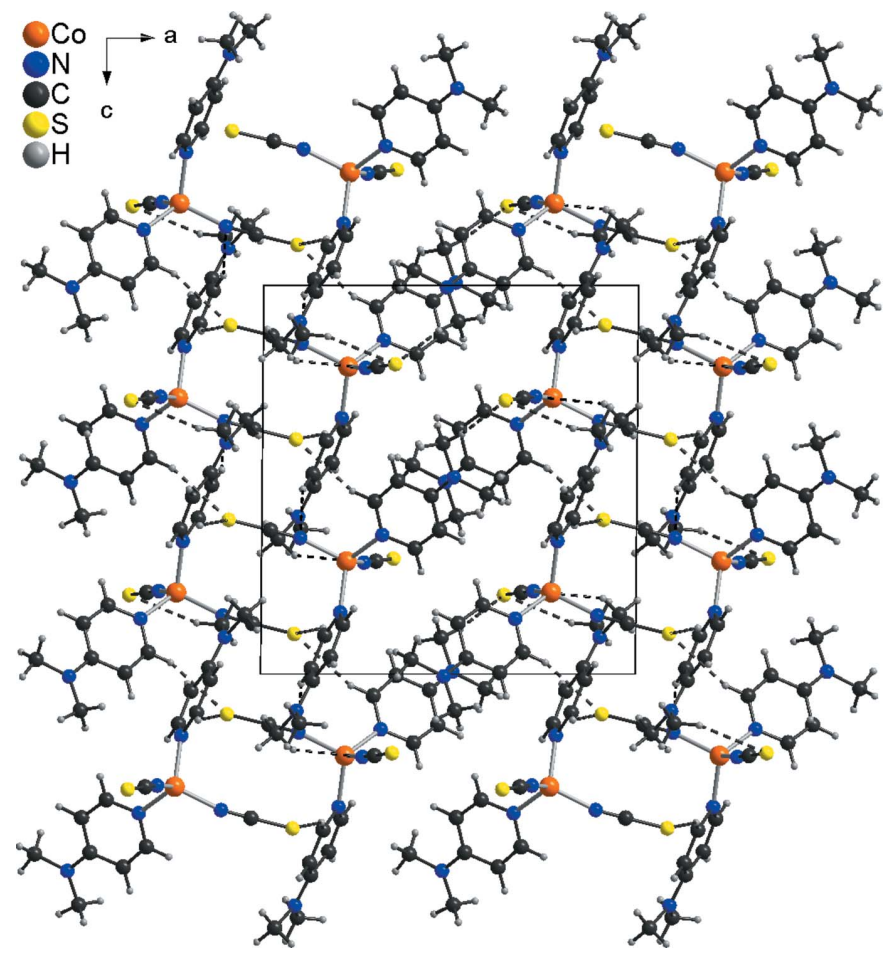

Figure 6

Crystal structure of form II with view in the direction of the crystallographic $b$-axis. Intermolecular $\mathrm{C}-\mathrm{H} \cdots \mathrm{S}$ and $\mathrm{C}-\mathrm{H} \cdots \mathrm{N}$ hydrogen bonds are shown as dashed lines. 
Table 5

Experimental details.

\begin{tabular}{|c|c|c|}
\hline & Form I & Form II \\
\hline \multicolumn{3}{|l|}{ Crystal data } \\
\hline Chemical formula & {$\left[\mathrm{Co}(\mathrm{NCS})_{2}\left(\mathrm{C}_{7} \mathrm{H}_{10} \mathrm{~N}_{2}\right)_{2}\right]$} & $\mathrm{C}_{16} \mathrm{H}_{20} \mathrm{CoN}_{6} \mathrm{~S}_{2}$ \\
\hline$M_{\mathrm{r}}$ & 419.43 & 419.43 \\
\hline Temperature (K) & 100 & 100 \\
\hline$a, b, c(\AA)$ & $5.3708(1), 15.2200(2), 11.8014(1)$ & $13.9171(1), 9.5114(1), 14.4487(1)$ \\
\hline$\beta\left(^{\circ}\right)$ & $99.076(1)$ & $90.489(1)$ \\
\hline$V\left(\AA^{3}\right)$ & $952.61(2)$ & $1912.52(3)$ \\
\hline$\mu\left(\mathrm{mm}^{-1}\right)$ & 9.20 & 9.17 \\
\hline Crystal size (mm) & $0.2 \times 0.12 \times 0.04$ & $0.18 \times 0.1 \times 0.03$ \\
\hline \multicolumn{3}{|l|}{ Data collection } \\
\hline Diffractometer & XtaLAB Synergy, Dualflex, HyPix & XtaLAB Synergy, Dualflex, HyPix \\
\hline Absorption correction & Multi-scan (CrysAlis PRO; Rigaku OD, 2021) & Multi-scan (CrysAlis PRO; Rigaku OD, 2021) \\
\hline$T_{\min }, T_{\max }$ & $0.311,1.000$ & $0.476,1.000$ \\
\hline \multicolumn{3}{|l|}{ Refinement } \\
\hline$R\left[F^{2}>2 \sigma\left(F^{2}\right)\right], w R\left(F^{2}\right), S$ & $0.024,0.067,1.14$ & $0.026,0.073,1.13$ \\
\hline No. of reflections & 2100 & 4151 \\
\hline No. of parameters & 126 & 231 \\
\hline $\mathrm{H}$-atom treatment & $\mathrm{H}$-atom parameters constrained & $\mathrm{H}$-atom parameters constrained \\
\hline$\Delta \rho_{\max }, \Delta \rho_{\min }\left(\mathrm{e} \AA^{-3}\right)$ & $0.25,-0.37$ & $0.29,-0.35$ \\
\hline
\end{tabular}

Computer programs: CrysAlis PRO (Rigaku OD, 2021), SHELXT2014/5 (Sheldrick, 2015a), SHELXL2016/6 (Sheldrick, 2015b), DIAMOND (Brandenburg \& Putz, 1999) and publCIF (Westrip, 2010).

\section{Synthesis and crystallization}

$\mathrm{Co}(\mathrm{NCS})_{2}$ and DMAP were purchased from Merck. All chemicals were used without further purification.

Blue single crystals of form I suitable for single crystal $\mathrm{X}$-ray analysis were obtained three days after storing $0.15 \mathrm{mmol} \mathrm{Co}(\mathrm{NCS})_{2} \quad(26.3 \mathrm{mg})$ and $0.30 \mathrm{mmol}$ DMAP (36.6 mg) in $1.0 \mathrm{ml} \mathrm{H}_{2} \mathrm{O}$ at $333 \mathrm{~K}$ followed by slow cooling.

Single crystals of form II were obtained as described in the literature (Neumann et al., 2018a).

\section{Refinement}

The $\mathrm{C}$-bound $\mathrm{H}$ atoms were located in the difference map but positioned with idealized geometry $(\mathrm{C}-\mathrm{H}=0.95-0.98 \AA$; methyl $\mathrm{H}$ atoms allowed to rotate but not to tip) and were refined isotropically with $U_{\text {iso }}(\mathrm{H})=1.2 U_{\text {eq }}(\mathrm{C})\left[1.5 U_{\text {eq }}(\mathrm{C})\right.$ for methyl $\mathrm{H}$ atoms] using a riding model. Crystal data, data collection and structure refinement details are summarized in Table 5.

\section{Acknowledgements}

Financial support by the State of Schleswig-Holstein is gratefully acknowledged.

\section{Funding information}

Funding for this research was provided by: Deutsche Forschungsgemeinschaft (grant No. NA720/5-2).

\section{References}

Barnett, S. A., Blake, A. J., Champness, N. R. \& Wilson, C. (2002). Chem. Commun. pp. 1640-1641.

Batten, Sr, R., Neville, S. M. \& Turner, D. R. (2009). Coordination Polymers: Design, Analysis and Application. Cambridge: The Royal Society of Chemistry.

Böhme, M., Jochim, A., Rams, M., Lohmiller, T., Suckert, S., Schnegg, A., Plass, W. \& Näther, C. (2020). Inorg. Chem. 59, 5325-5338.

Braga, D. \& Grepioni, F. (2000). Chem. Soc. Rev. 29, 229-238.

Braga, D., Grepioni, F. \& Desiraju, G. (1998). Chem. Rev. 98, 13751406.

Brandenburg, K. \& Putz, H. (1999). DIAMOND. Crystal Impact GbR, Bonn, Germany.

Buckingham, D. A. (1994). Coord. Chem. Rev. 135-136, 587-621.

Burger, A. \& Ramberger, R. (1979). Mikrochim. Acta, 72, 259-271.

Chen, F., Liu, G. \& Zeng, Z. (2007). Anal. Sci. X, 23, X253-X254.

Jochim, A., Rams, M., Böhme, M., Ceglarska, M., Plass, W. \& Näther, C. (2020). Dalton Trans. 49, 15310-15322.

Jochim, A., Rams, M., Neumann, T., Wellm, C., Reinsch, H., Wójtowicz, G. M. \& Näther, C. (2018). Eur. J. Inorg. Chem. pp. 4779-4789.

Kitaigorodskii, A. I. (1961). Organic Chemical Crystallography. New York.: Consultants Bureau,

Mautner, F. A., Traber, M., Fischer, R. C., Torvisco, A., Reichmann, K., Speed, S., Vicente, R. \& Massoud, S. S. (2018). Polyhedron, 154, 436-442.

Moulton, B. \& Zaworotko, M. J. (2001). Chem. Rev. 101, 1629-1658.

Neumann, T., Gallo, G., Jess, I., Dinnebier, R. E. \& Näther, C. (2020a). CrystEngComm, 22, 184-194.

Neumann, T., Jess, I., German, L. S., Dinnebier, R. E. \& Näther, C. (2020b). Cryst. Growth Des. 19, 1143-1143.

Neumann, T., Jess, I., Pielnhofer, F. \& Näther, C. (2018a). Eur. J. Inorg. Chem. pp. 4972-4981. 
Neumann, T., Rams, M., Wellm, C. \& Näther, C. (2018b). Cryst. Growth Des. 18, 6020-6027.

Ossinger, S., Näther, C., Buchholz, A., Schmidtmann, M., Mangelsen, S., Beckhaus, R., Plass, W. \& Tuczek, F. (2020). Inorg. Chem. 59, 7966-7979.

Rigaku OD (2021). CrysAlis PRO. Rigaku Oxford Diffraction.

Secondo, P. M., Land, J. M., Baughman, R. G. \& Collier, H. L. (2000). Inorg. Chim. Acta, 309, 13-22.

Sheldrick, G. M. (2015a). Acta Cryst. A71, 3-8.

Sheldrick, G. M. (2015b). Acta Cryst. C71, 3-8.

Sheu, C. F., Chen, K., Chen, S. M., Wen, Y. S., Lee, G. H., Chen, J. M., Lee, J. F., Cheng, B. M., Sheu, H. S., Yasuda, N., Ozawa, Y., Toriumi, K. \& Wang, Y. (2009). Chem. Eur. J. 15, 2384-2393.

Suckert, S., Jess, I. \& Näther, C. (2015). Acta Cryst. E71, m126.
Tao, J., Wei, R. J., Huang, R. B. \& Zheng, L. S. (2012). Chem. Soc. Rev. 41, 703-737.

Wellm, C., Majcher-Fitas, A., Rams, M. \& Näther, C. (2020b). Dalton Trans. 49, 16707-16714.

Wellm, C., Neumann, T., Gallo, G., Dziubyna, A. M., Rams, M., Dinnebier, R. E. \& Näther, C. (2020a). Cryst. Growth Des. 20, 3374-3385.

Wellm, C., Rams, M., Neumann, T., Ceglarska, M. \& Näther, C. (2018). Cryst. Growth Des. 18, 3117-3123.

Werner, J., Runčevski, T., Dinnebier, R. E., Ebbinghaus, S. G., Suckert, S. \& Näther, C. (2015). Eur. J. Inorg. Chem. pp. 3236-3245. Westrip, S. P. (2010). J. Appl. Cryst. 43, 920-925.

Zhang, J. P., Huang, X. C. \& Chen, X. M. (2009). Chem. Soc. Rev. 38, 2385-2396. 


\section{supporting information}

Acta Cryst. (2021). E77, 1120-1125 [https://doi.org/10.1107/S2056989021010422]

Comparison of the crystal structures of the low- and high-temperature forms of bis[4-(dimethylamino)pyridine] dithiocyanatocobalt(II)

\section{Christoph Krebs, Inke Jess and Christian Näther}

Computing details

For both structures, data collection: CrysAlis PRO (Rigaku OD, 2021); cell refinement: CrysAlis PRO (Rigaku OD, 2021); data reduction: CrysAlis PRO (Rigaku OD, 2021); program(s) used to solve structure: SHELXT2014/5 (Sheldrick, 2015a); program(s) used to refine structure: SHELXL2016/6 (Sheldrick, 2015b); molecular graphics: DIAMOND

(Brandenburg \& Putz, 1999); software used to prepare material for publication: publCIF (Westrip, 2010).

Bis[4-(dimethylamino)pyridine] dithiocyanatocobalt(II) (Form_I)

\section{Crystal data}

$\left[\mathrm{Co}(\mathrm{NCS})_{2}\left(\mathrm{C}_{7} \mathrm{H}_{10} \mathrm{~N}_{2}\right)_{2}\right]$

$M_{r}=419.43$

Monoclinic, $P 12_{1} / m 1$

$a=5.3708(1) \AA$

$b=15.2200(2) \AA$

$c=11.8014(1) \AA$

$\beta=99.076(1)^{\circ}$

$V=952.61(2) \AA^{3}$

$Z=2$

\section{Data collection}

XtaLAB Synergy, Dualflex, HyPix diffractometer

Radiation source: micro-focus sealed X-ray tube, PhotonJet $(\mathrm{Cu}) \mathrm{X}$-ray Source

Mirror monochromator

Detector resolution: 10.0000 pixels $\mathrm{mm}^{-1}$

$\omega$ scans

Absorption correction: multi-scan

(CrysalisPro; Rigaku OD, 2021)

\section{Refinement}

Refinement on $F^{2}$

Least-squares matrix: full

$R\left[F^{2}>2 \sigma\left(F^{2}\right)\right]=0.024$

$w R\left(F^{2}\right)=0.067$

$S=1.14$

2100 reflections

126 parameters

0 restraints

Primary atom site location: dual
$F(000)=434$

$D_{\mathrm{x}}=1.462 \mathrm{Mg} \mathrm{m}^{-3}$

$\mathrm{Cu} K \alpha$ radiation, $\lambda=1.54184 \AA$

Cell parameters from 13880 reflections

$\theta=3.8-77.3^{\circ}$

$\mu=9.20 \mathrm{~mm}^{-1}$

$T=100 \mathrm{~K}$

Block, light blue

$0.2 \times 0.12 \times 0.04 \mathrm{~mm}$

$T_{\min }=0.311, T_{\max }=1.000$

16411 measured reflections

2100 independent reflections

2081 reflections with $I>2 \sigma(I)$

$R_{\text {int }}=0.025$

$\theta_{\max }=78.1^{\circ}, \theta_{\min }=3.8^{\circ}$

$h=-6 \rightarrow 5$

$k=-19 \rightarrow 19$

$l=-14 \rightarrow 14$

Hydrogen site location: inferred from neighbouring sites

$\mathrm{H}$-atom parameters constrained

$w=1 /\left[\sigma^{2}\left(F_{\mathrm{o}}^{2}\right)+(0.0348 P)^{2}+0.4033 P\right]$

where $P=\left(F_{\mathrm{o}}^{2}+2 F_{\mathrm{c}}^{2}\right) / 3$

$(\Delta / \sigma)_{\max }=0.001$

$\Delta \rho_{\max }=0.25$ e $\AA^{-3}$

$\Delta \rho_{\min }=-0.37$ e $\AA^{-3}$ 


\section{Special details}

Geometry. All esds (except the esd in the dihedral angle between two 1.s. planes) are estimated using the full covariance matrix. The cell esds are taken into account individually in the estimation of esds in distances, angles and torsion angles; correlations between esds in cell parameters are only used when they are defined by crystal symmetry. An approximate (isotropic) treatment of cell esds is used for estimating esds involving l.s. planes.

Fractional atomic coordinates and isotropic or equivalent isotropic displacement parameters $\left(\AA^{2}\right)$

\begin{tabular}{lllll}
\hline & $x$ & $y$ & $z$ & $U_{\text {iso }} / U_{\text {eq }}$ \\
\hline Co1 & $0.93113(6)$ & 0.750000 & $0.27609(3)$ & $0.01820(10)$ \\
N1 & $1.1349(3)$ & 0.750000 & $0.15392(15)$ & $0.0236(4)$ \\
C1 & $1.2586(4)$ & 0.750000 & $0.08142(17)$ & $0.0197(4)$ \\
S1 & $1.43347(10)$ & 0.750000 & $-0.01905(4)$ & $0.02516(13)$ \\
N2 & $1.1055(3)$ & 0.750000 & $0.43574(15)$ & $0.0228(4)$ \\
C2 & $1.2525(4)$ & 0.750000 & $0.52018(17)$ & $0.0202(4)$ \\
S2 & $1.46184(10)$ & 0.750000 & $0.63528(4)$ & $0.02618(13)$ \\
N11 & $0.7113(2)$ & $0.64220(8)$ & $0.26788(10)$ & $0.0186(2)$ \\
C11 & $0.5802(3)$ & $0.62788(9)$ & $0.35490(12)$ & $0.0194(3)$ \\
H11 & 0.614076 & 0.664879 & 0.420344 & $0.023^{*}$ \\
C12 & $0.4021(3)$ & $0.56372(9)$ & $0.35509(12)$ & $0.0197(3)$ \\
H12 & 0.318372 & 0.556780 & 0.419671 & $0.024^{*}$ \\
C13 & $0.3428(3)$ & $0.50769(9)$ & $0.25904(12)$ & $0.0196(3)$ \\
C14 & $0.4861(3)$ & $0.52099(10)$ & $0.16919(12)$ & $0.0216(3)$ \\
H14 & 0.460807 & 0.483952 & 0.103587 & $0.026^{*}$ \\
C15 & $0.6609(3)$ & $0.58727(9)$ & $0.17704(12)$ & $0.0203(3)$ \\
H15 & 0.752119 & 0.595055 & 0.115020 & $0.024^{*}$ \\
N12 & $0.1612(2)$ & $0.44622(8)$ & $0.25328(11)$ & $0.0229(3)$ \\
C16 & $0.0279(3)$ & $0.43142(10)$ & $0.34987(14)$ & $0.0265(3)$ \\
H16A & 0.147210 & 0.410913 & 0.416077 & $0.040^{*}$ \\
H16B & -0.103220 & 0.386937 & 0.329058 & $0.040^{*}$ \\
H16C & -0.049866 & 0.486459 & 0.369491 & $0.040^{*}$ \\
C17 & $0.1036(3)$ & $0.38967(11)$ & $0.15329(15)$ & $0.0320(4)$ \\
H17A & 0.053675 & 0.425800 & 0.084731 & $0.048^{*}$ \\
H17B & -0.034880 & 0.349998 & 0.163508 & $0.048^{*}$ \\
H17C & 0.253102 & 0.355095 & 0.144230 & $0.048^{*}$ \\
& & & & \\
\hline
\end{tabular}

Atomic displacement parameters $\left(\AA^{2}\right)$

\begin{tabular}{lllllll}
\hline & $U^{11}$ & $U^{22}$ & $U^{33}$ & $U^{12}$ & $U^{13}$ & $U^{23}$ \\
\hline Co1 & $0.01752(17)$ & $0.01727(17)$ & $0.02091(17)$ & 0.000 & $0.00643(12)$ & 0.000 \\
N1 & $0.0219(8)$ & $0.0256(9)$ & $0.0246(9)$ & 0.000 & $0.0077(7)$ & 0.000 \\
C1 & $0.0170(9)$ & $0.0193(9)$ & $0.0220(9)$ & 0.000 & $0.0007(7)$ & 0.000 \\
S1 & $0.0251(3)$ & $0.0307(3)$ & $0.0215(2)$ & 0.000 & $0.00917(19)$ & 0.000 \\
N2 & $0.0225(8)$ & $0.0226(9)$ & $0.0246(9)$ & 0.000 & $0.0076(7)$ & 0.000 \\
C2 & $0.0223(9)$ & $0.0160(9)$ & $0.0248(10)$ & 0.000 & $0.0108(8)$ & 0.000 \\
S2 & $0.0249(3)$ & $0.0292(3)$ & $0.0237(2)$ & 0.000 & $0.00158(19)$ & 0.000 \\
N11 & $0.0187(5)$ & $0.0172(5)$ & $0.0203(5)$ & $0.0012(4)$ & $0.0043(4)$ & $0.0012(4)$ \\
C11 & $0.0211(6)$ & $0.0187(6)$ & $0.0190(6)$ & $0.0022(5)$ & $0.0047(5)$ & $-0.0010(5)$
\end{tabular}


supporting information

\begin{tabular}{lllllll}
$\mathrm{C} 12$ & $0.0218(6)$ & $0.0195(7)$ & $0.0191(6)$ & $0.0030(5)$ & $0.0071(5)$ & $0.0015(5)$ \\
$\mathrm{C} 13$ & $0.0179(6)$ & $0.0179(6)$ & $0.0229(6)$ & $0.0021(5)$ & $0.0027(5)$ & $0.0015(5)$ \\
$\mathrm{C} 14$ & $0.0242(7)$ & $0.0221(7)$ & $0.0187(6)$ & $0.0011(6)$ & $0.0041(5)$ & $-0.0030(5)$ \\
$\mathrm{C} 15$ & $0.0220(6)$ & $0.0217(7)$ & $0.0181(6)$ & $0.0023(6)$ & $0.0062(5)$ & $0.0003(5)$ \\
$\mathrm{N} 12$ & $0.0222(6)$ & $0.0196(6)$ & $0.0275(6)$ & $-0.0022(5)$ & $0.0055(5)$ & $-0.0016(5)$ \\
$\mathrm{C} 16$ & $0.0234(7)$ & $0.0246(7)$ & $0.0324(8)$ & $-0.0020(6)$ & $0.0067(6)$ & $0.0059(6)$ \\
$\mathrm{C} 17$ & $0.0302(8)$ & $0.0280(8)$ & $0.0379(9)$ & $-0.0072(7)$ & $0.0056(7)$ & $-0.0099(7)$ \\
\hline
\end{tabular}

Geometric parameters $\left(A,{ }^{\circ}\right)$

\begin{tabular}{|c|c|c|c|}
\hline $\mathrm{Co} 1-\mathrm{N} 1$ & $1.9429(18)$ & $\mathrm{C} 13-\mathrm{C} 14$ & $1.4195(19)$ \\
\hline $\mathrm{Co} 1-\mathrm{N} 2$ & $1.9672(19)$ & $\mathrm{C} 13-\mathrm{N} 12$ & $1.3454(19)$ \\
\hline $\mathrm{Co} 1-\mathrm{N} 11$ & $2.0148(12)$ & $\mathrm{C} 14-\mathrm{H} 14$ & 0.9500 \\
\hline $\mathrm{Co} 1-\mathrm{N} 11^{\mathrm{i}}$ & $2.0148(12)$ & $\mathrm{C} 14-\mathrm{C} 15$ & $1.371(2)$ \\
\hline $\mathrm{N} 1-\mathrm{C} 1$ & $1.163(3)$ & $\mathrm{C} 15-\mathrm{H} 15$ & 0.9500 \\
\hline $\mathrm{C} 1-\mathrm{S} 1$ & $1.625(2)$ & $\mathrm{N} 12-\mathrm{C} 16$ & $1.4559(19)$ \\
\hline $\mathrm{N} 2-\mathrm{C} 2$ & $1.170(3)$ & $\mathrm{N} 12-\mathrm{C} 17$ & $1.454(2)$ \\
\hline $\mathrm{C} 2-\mathrm{S} 2$ & $1.621(2)$ & $\mathrm{C} 16-\mathrm{H} 16 \mathrm{~A}$ & 0.9800 \\
\hline $\mathrm{N} 11-\mathrm{C} 11$ & $1.3512(17)$ & C16-H16B & 0.9800 \\
\hline $\mathrm{N} 11-\mathrm{C} 15$ & $1.3529(18)$ & $\mathrm{C} 16-\mathrm{H} 16 \mathrm{C}$ & 0.9800 \\
\hline C11-H11 & 0.9500 & C17-H17A & 0.9800 \\
\hline $\mathrm{C} 11-\mathrm{C} 12$ & $1.367(2)$ & $\mathrm{C} 17-\mathrm{H} 17 \mathrm{~B}$ & 0.9800 \\
\hline $\mathrm{C} 12-\mathrm{H} 12$ & 0.9500 & $\mathrm{C} 17-\mathrm{H} 17 \mathrm{C}$ & 0.9800 \\
\hline $\mathrm{C} 12-\mathrm{C} 13$ & $1.414(2)$ & & \\
\hline $\mathrm{N} 1-\mathrm{Co} 1-\mathrm{N} 2$ & $118.16(8)$ & $\mathrm{C} 13-\mathrm{C} 14-\mathrm{H} 14$ & 120.0 \\
\hline $\mathrm{N} 1-\mathrm{Co} 1-\mathrm{N} 11$ & $111.03(4)$ & $\mathrm{C} 15-\mathrm{C} 14-\mathrm{C} 13$ & $120.05(13)$ \\
\hline $\mathrm{N} 1-\mathrm{Co} 1-\mathrm{N} 11^{\mathrm{i}}$ & $111.03(4)$ & $\mathrm{C} 15-\mathrm{C} 14-\mathrm{H} 14$ & 120.0 \\
\hline $\mathrm{N} 2-\mathrm{Co} 1-\mathrm{N} 11$ & $103.47(4)$ & $\mathrm{N} 11-\mathrm{C} 15-\mathrm{C} 14$ & $123.86(13)$ \\
\hline $\mathrm{N} 2-\mathrm{Co} 1-\mathrm{N} 11^{\mathrm{i}}$ & $103.47(4)$ & $\mathrm{N} 11-\mathrm{C} 15-\mathrm{H} 15$ & 118.1 \\
\hline $\mathrm{N} 11-\mathrm{Co} 1-\mathrm{N} 11^{\mathrm{i}}$ & $109.04(7)$ & $\mathrm{C} 14-\mathrm{C} 15-\mathrm{H} 15$ & 118.1 \\
\hline $\mathrm{C} 1-\mathrm{N} 1-\mathrm{Co} 1$ & $179.48(17)$ & $\mathrm{C} 13-\mathrm{N} 12-\mathrm{C} 16$ & $120.66(12)$ \\
\hline $\mathrm{N} 1-\mathrm{C} 1-\mathrm{S} 1$ & $179.51(19)$ & $\mathrm{C} 13-\mathrm{N} 12-\mathrm{C} 17$ & $120.83(13)$ \\
\hline $\mathrm{C} 2-\mathrm{N} 2-\mathrm{Co} 1$ & $166.24(16)$ & $\mathrm{C} 17-\mathrm{N} 12-\mathrm{C} 16$ & $118.42(12)$ \\
\hline $\mathrm{N} 2-\mathrm{C} 2-\mathrm{S} 2$ & $178.57(18)$ & $\mathrm{N} 12-\mathrm{C} 16-\mathrm{H} 16 \mathrm{~A}$ & 109.5 \\
\hline $\mathrm{C} 11-\mathrm{N} 11-\mathrm{Co} 1$ & $117.81(9)$ & $\mathrm{N} 12-\mathrm{C} 16-\mathrm{H} 16 \mathrm{~B}$ & 109.5 \\
\hline $\mathrm{C} 11-\mathrm{N} 11-\mathrm{C} 15$ & $116.08(12)$ & $\mathrm{N} 12-\mathrm{C} 16-\mathrm{H} 16 \mathrm{C}$ & 109.5 \\
\hline $\mathrm{C} 15-\mathrm{N} 11-\mathrm{Co} 1$ & $125.71(9)$ & $\mathrm{H} 16 \mathrm{~A}-\mathrm{C} 16-\mathrm{H} 16 \mathrm{~B}$ & 109.5 \\
\hline N11-C11-H11 & 117.8 & $\mathrm{H} 16 \mathrm{~A}-\mathrm{C} 16-\mathrm{H} 16 \mathrm{C}$ & 109.5 \\
\hline $\mathrm{N} 11-\mathrm{C} 11-\mathrm{C} 12$ & $124.41(13)$ & $\mathrm{H} 16 \mathrm{~B}-\mathrm{C} 16-\mathrm{H} 16 \mathrm{C}$ & 109.5 \\
\hline $\mathrm{C} 12-\mathrm{C} 11-\mathrm{H} 11$ & 117.8 & $\mathrm{~N} 12-\mathrm{C} 17-\mathrm{H} 17 \mathrm{~A}$ & 109.5 \\
\hline $\mathrm{C} 11-\mathrm{C} 12-\mathrm{H} 12$ & 120.0 & N12-C17-H17B & 109.5 \\
\hline $\mathrm{C} 11-\mathrm{C} 12-\mathrm{C} 13$ & $119.91(13)$ & $\mathrm{N} 12-\mathrm{C} 17-\mathrm{H} 17 \mathrm{C}$ & 109.5 \\
\hline $\mathrm{C} 13-\mathrm{C} 12-\mathrm{H} 12$ & 120.0 & $\mathrm{H} 17 \mathrm{~A}-\mathrm{C} 17-\mathrm{H} 17 \mathrm{~B}$ & 109.5 \\
\hline $\mathrm{C} 12-\mathrm{C} 13-\mathrm{C} 14$ & $115.63(13)$ & $\mathrm{H} 17 \mathrm{~A}-\mathrm{C} 17-\mathrm{H} 17 \mathrm{C}$ & 109.5 \\
\hline
\end{tabular}


$\mathrm{N} 12-\mathrm{C} 13-\mathrm{C} 12$

$\mathrm{N} 12-\mathrm{C} 13-\mathrm{C} 14$
$121.99(13)$

$122.38(13)$

H17B-C17-H17C

109.5

Symmetry code: (i) $x,-y+3 / 2, z$.

Hydrogen-bond geometry $\left(A,{ }^{\circ}\right)$

\begin{tabular}{lllll}
\hline$D-\mathrm{H} \cdots A$ & $D-\mathrm{H}$ & $\mathrm{H} \cdots A$ & $D \cdots A$ & $D-\mathrm{H} \cdots A$ \\
\hline $\mathrm{C} 16-\mathrm{H} 16 B \cdots \mathrm{S} 2^{\mathrm{ii}}$ & 0.98 & 2.91 & $3.8367(15)$ & 158 \\
$\mathrm{C} 17-\mathrm{H} 17 B^{\mathrm{ii}} \mathrm{S} 1^{\mathrm{iii}}$ & 0.98 & 2.94 & $3.7291(16)$ & 138 \\
$\mathrm{C} 17-\mathrm{H} 17 C \cdots \mathrm{S} 1^{\mathrm{iv}}$ & 0.98 & 2.89 & $3.8018(18)$ & 155
\end{tabular}

Symmetry codes: (ii) $-x+1,-y+1,-z+1$; (iii) $-x+1,-y+1,-z$; (iv) $-x+2,-y+1,-z$.

(Form_II)

Crystal data

$\mathrm{C}_{16} \mathrm{H}_{20} \mathrm{CoN}_{6} \mathrm{~S}_{2}$

$M_{r}=419.43$

Monoclinic, $P 2{ }_{1} / c$

$a=13.9171$ (1) $\AA$

$b=9.5114(1) \AA$

$c=14.4487$ (1) $\AA$

$\beta=90.489(1)^{\circ}$

$V=1912.52(3) \AA^{3}$

$Z=4$

$F(000)=868$

$D_{\mathrm{x}}=1.457 \mathrm{Mg} \mathrm{m}^{-3}$

$\mathrm{Cu} K \alpha$ radiation, $\lambda=1.54184 \AA$

Cell parameters from 41675 reflections

$\theta=3.2-79.4^{\circ}$

$\mu=9.17 \mathrm{~mm}^{-1}$

$T=100 \mathrm{~K}$

Block, dark blue

$0.18 \times 0.1 \times 0.03 \mathrm{~mm}$

\section{Data collection}

XtaLAB Synergy, Dualflex, HyPix diffractometer

Radiation source: micro-focus sealed X-ray tube, PhotonJet $(\mathrm{Cu}) \mathrm{X}$-ray Source

Mirror monochromator

Detector resolution: 10.0000 pixels $\mathrm{mm}^{-1}$

$\omega$ scans

Absorption correction: multi-scan

(CrysalisPro; Rigaku OD, 2021)

$T_{\min }=0.476, T_{\max }=1.000$

57319 measured reflections

4151 independent reflections

4079 reflections with $I>2 \sigma(I)$

$R_{\text {int }}=0.025$

$\theta_{\max }=80.2^{\circ}, \theta_{\min }=3.2^{\circ}$

$h=-17 \rightarrow 17$

$k=-12 \rightarrow 12$

$l=-18 \rightarrow 18$

\section{Refinement}

Refinement on $F^{2}$

Least-squares matrix: full

$R\left[F^{2}>2 \sigma\left(F^{2}\right)\right]=0.026$

$w R\left(F^{2}\right)=0.073$

$S=1.13$

4151 reflections

231 parameters

0 restraints

Primary atom site location: dual

Hydrogen site location: inferred from neighbouring sites

$\mathrm{H}$-atom parameters constrained

$w=1 /\left[\sigma^{2}\left(F_{\mathrm{o}}{ }^{2}\right)+(0.0406 P)^{2}+0.8179 P\right]$

where $P=\left(F_{\mathrm{o}}^{2}+2 F_{\mathrm{c}}^{2}\right) / 3$

$(\Delta / \sigma)_{\max }=0.002$

$\Delta \rho_{\max }=0.29 \mathrm{e} \AA^{-3}$

$\Delta \rho_{\min }=-0.35$ e $\AA^{-3}$

Extinction correction: SHELXL2016/6

(Sheldrick, 2015b),

$\mathrm{Fc}^{*}=\mathrm{kFc}\left[1+0.001 \mathrm{xFc}^{2} \lambda^{3} / \sin (2 \theta)\right]^{-1 / 4}$

Extinction coefficient: 0.00076 (11) 


\section{Special details}

Geometry. All esds (except the esd in the dihedral angle between two 1.s. planes) are estimated using the full covariance matrix. The cell esds are taken into account individually in the estimation of esds in distances, angles and torsion angles; correlations between esds in cell parameters are only used when they are defined by crystal symmetry. An approximate (isotropic) treatment of cell esds is used for estimating esds involving l.s. planes.

Fractional atomic coordinates and isotropic or equivalent isotropic displacement parameters $\left(\AA^{2}\right)$

\begin{tabular}{|c|c|c|c|c|}
\hline & $x$ & $y$ & $z$ & $U_{\text {iso }} * / U_{\text {eq }}$ \\
\hline Col & $0.77328(2)$ & $0.36737(2)$ & $0.79003(2)$ & $0.01802(8)$ \\
\hline N1 & $0.89733(9)$ & $0.40269(14)$ & $0.84966(9)$ & $0.0228(3)$ \\
\hline $\mathrm{C} 1$ & $0.97734(11)$ & $0.42519(15)$ & $0.87028(9)$ & $0.0203(3)$ \\
\hline $\mathrm{S} 1$ & $1.08865(3)$ & $0.45439(4)$ & $0.89675(3)$ & $0.02797(10)$ \\
\hline N2 & $0.72639(9)$ & $0.17383(14)$ & $0.78687(9)$ & 0.0249 (3) \\
\hline $\mathrm{C} 2$ & $0.69467(10)$ & $0.06052(16)$ & $0.79023(10)$ & $0.0225(3)$ \\
\hline $\mathrm{S} 2$ & $0.64780(3)$ & -0.09564 (4) & $0.79675(3)$ & $0.02997(10)$ \\
\hline N11 & $0.67807(8)$ & $0.48667(13)$ & $0.85774(8)$ & $0.0203(2)$ \\
\hline C11 & $0.69896(10)$ & $0.53200(16)$ & $0.94415(10)$ & $0.0213(3)$ \\
\hline H11 & 0.759338 & 0.506648 & 0.970392 & $0.026^{*}$ \\
\hline $\mathrm{C} 12$ & $0.63811(11)$ & $0.61250(16)$ & $0.99628(10)$ & $0.0218(3)$ \\
\hline H12 & 0.656100 & 0.639410 & 1.057333 & $0.026^{*}$ \\
\hline $\mathrm{C} 13$ & $0.54861(10)$ & $0.65531(15)$ & $0.95897(10)$ & 0.0199 (3) \\
\hline C14 & $0.52571(10)$ & $0.60389(16)$ & $0.86934(10)$ & $0.0222(3)$ \\
\hline H14 & 0.465517 & 0.625731 & 0.841373 & $0.027^{*}$ \\
\hline $\mathrm{C} 15$ & $0.59106(10)$ & $0.52243(16)$ & $0.82332(10)$ & $0.0213(3)$ \\
\hline H15 & 0.573977 & 0.489080 & 0.763427 & $0.026^{*}$ \\
\hline N12 & $0.48862(9)$ & $0.74021(14)$ & $1.00607(9)$ & $0.0241(3)$ \\
\hline $\mathrm{C} 16$ & $0.51936(12)$ & 0.80309 (19) & $1.09350(12)$ & $0.0303(3)$ \\
\hline H16A & 0.577275 & 0.859533 & 1.083572 & $0.045^{*}$ \\
\hline H16B & 0.533416 & 0.728603 & 1.138480 & $0.045^{*}$ \\
\hline $\mathrm{H} 16 \mathrm{C}$ & 0.468041 & 0.863388 & 1.117198 & $0.045^{*}$ \\
\hline $\mathrm{C} 17$ & $0.40047(11)$ & $0.79162(19)$ & $0.96272(12)$ & $0.0309(3)$ \\
\hline H17A & 0.360713 & 0.711636 & 0.943368 & $0.046^{*}$ \\
\hline H17B & 0.416451 & 0.848679 & 0.908512 & $0.046^{*}$ \\
\hline $\mathrm{H} 17 \mathrm{C}$ & 0.364953 & 0.849121 & 1.007120 & $0.046^{*}$ \\
\hline N21 & $0.78919(8)$ & $0.42769(13)$ & $0.65835(8)$ & $0.0189(2)$ \\
\hline $\mathrm{C} 21$ & $0.82983(10)$ & $0.33442(16)$ & $0.59926(10)$ & $0.0196(3)$ \\
\hline H21 & 0.834119 & 0.238949 & 0.618167 & $0.024^{*}$ \\
\hline $\mathrm{C} 22$ & $0.86504(11)$ & $0.36954(15)$ & $0.51404(10)$ & $0.0209(3)$ \\
\hline H22 & 0.891632 & 0.299061 & 0.475335 & $0.025^{*}$ \\
\hline $\mathrm{C} 23$ & $0.86172(10)$ & $0.51100(16)$ & $0.48379(9)$ & $0.0200(3)$ \\
\hline $\mathrm{C} 24$ & $0.81437(11)$ & $0.60652(16)$ & $0.54359(11)$ & $0.0227(3)$ \\
\hline H24 & 0.805027 & 0.701424 & 0.525107 & $0.027^{*}$ \\
\hline $\mathrm{C} 25$ & $0.78214(10)$ & $0.56180(15)$ & $0.62799(10)$ & $0.0211(3)$ \\
\hline H25 & 0.753116 & 0.629049 & 0.667518 & $0.025^{*}$ \\
\hline N22 & $0.90343(9)$ & $0.55389(14)$ & $0.40487(8)$ & $0.0231(3)$ \\
\hline $\mathrm{C} 26$ & $0.95644(11)$ & $0.45537(17)$ & $0.34714(10)$ & $0.0248(3)$ \\
\hline H26A & 0.911620 & 0.406459 & 0.305536 & $0.037 *$ \\
\hline
\end{tabular}




$\begin{array}{lllll}\mathrm{H} 26 \mathrm{~B} & 0.989513 & 0.386597 & 0.386600 & 0.037^{*} \\ \mathrm{H} 26 \mathrm{C} & 1.003783 & 0.506881 & 0.310500 & 0.037^{*} \\ \mathrm{C} 27 & 0.89616(12) & 0.69988(18) & 0.37410(11) & 0.0290(3) \\ \mathrm{H} 27 \mathrm{~A} & 0.927850 & 0.761294 & 0.419469 & 0.043^{*} \\ \mathrm{H} 27 \mathrm{~B} & 0.828308 & 0.726211 & 0.368232 & 0.043^{*} \\ \mathrm{H} 27 \mathrm{C} & 0.927463 & 0.710043 & 0.313971 & 0.043^{*}\end{array}$

Atomic displacement parameters $\left(\AA^{2}\right)$

\begin{tabular}{|c|c|c|c|c|c|c|}
\hline & $U^{11}$ & $U^{22}$ & $U^{33}$ & $U^{12}$ & $U^{13}$ & $U^{23}$ \\
\hline Co1 & $0.01610(13)$ & $0.02028(13)$ & $0.01770(13)$ & $-0.00148(8)$ & $0.00179(9)$ & $-0.00024(8)$ \\
\hline N1 & $0.0219(6)$ & $0.0263(6)$ & $0.0202(6)$ & $-0.0016(5)$ & $0.0008(5)$ & $0.0020(5)$ \\
\hline $\mathrm{C} 1$ & $0.0247(7)$ & $0.0197(7)$ & $0.0166(6)$ & $-0.0006(5)$ & $0.0008(5)$ & $0.0013(5)$ \\
\hline $\mathrm{S} 1$ & $0.02121(18)$ & $0.0317(2)$ & 0.03093 (19) & $-0.00319(14)$ & $-0.00599(14)$ & $0.00401(15)$ \\
\hline N2 & $0.0239(6)$ & $0.0224(6)$ & $0.0284(6)$ & $-0.0016(5)$ & $0.0022(5)$ & $0.0000(5)$ \\
\hline $\mathrm{C} 2$ & $0.0175(6)$ & $0.0262(8)$ & $0.0238(7)$ & $0.0019(6)$ & $-0.0009(5)$ & 0.0005 (6) \\
\hline $\mathrm{S} 2$ & $0.02535(19)$ & 0.02148 (19) & $0.0430(2)$ & $-0.00320(14)$ & $-0.00451(16)$ & $0.00357(16)$ \\
\hline N11 & $0.0187(6)$ & $0.0227(6)$ & $0.0196(5)$ & $-0.0013(5)$ & $0.0015(4)$ & $-0.0004(5)$ \\
\hline $\mathrm{C} 11$ & $0.0166(6)$ & $0.0262(7)$ & $0.0211(6)$ & $-0.0004(5)$ & $-0.0011(5)$ & $-0.0012(6)$ \\
\hline $\mathrm{C} 12$ & $0.0195(7)$ & $0.0248(7)$ & $0.0210(7)$ & $-0.0013(5)$ & $-0.0012(5)$ & $-0.0016(5)$ \\
\hline $\mathrm{C} 13$ & $0.0170(6)$ & $0.0193(6)$ & $0.0235(7)$ & $-0.0026(5)$ & $0.0022(5)$ & $0.0020(5)$ \\
\hline $\mathrm{C} 14$ & $0.0170(6)$ & $0.0259(7)$ & $0.0236(7)$ & $-0.0009(5)$ & $-0.0034(5)$ & $0.0031(6)$ \\
\hline $\mathrm{C} 15$ & $0.0210(7)$ & $0.0243(7)$ & $0.0187(6)$ & $-0.0019(5)$ & $-0.0015(5)$ & $0.0002(5)$ \\
\hline N12 & $0.0187(6)$ & $0.0264(6)$ & $0.0270(6)$ & $0.0034(5)$ & $0.0012(5)$ & $-0.0015(5)$ \\
\hline $\mathrm{C} 16$ & $0.0245(7)$ & $0.0333(9)$ & $0.0332(8)$ & $0.0030(6)$ & $0.0030(6)$ & $-0.0107(7)$ \\
\hline $\mathrm{C} 17$ & $0.0224(7)$ & $0.0324(8)$ & $0.0378(8)$ & 0.0089 (6) & $-0.0004(6)$ & $0.0007(7)$ \\
\hline N21 & $0.0170(5)$ & $0.0212(6)$ & $0.0185(5)$ & $-0.0003(4)$ & $0.0010(4)$ & $-0.0005(4)$ \\
\hline $\mathrm{C} 21$ & $0.0188(6)$ & $0.0193(6)$ & $0.0207(6)$ & $-0.0009(5)$ & $0.0007(5)$ & $-0.0015(5)$ \\
\hline $\mathrm{C} 22$ & $0.0183(7)$ & $0.0241(7)$ & $0.0203(7)$ & $-0.0007(5)$ & $0.0003(5)$ & $-0.0034(5)$ \\
\hline $\mathrm{C} 23$ & $0.0153(6)$ & $0.0253(7)$ & $0.0194(6)$ & $-0.0031(5)$ & $-0.0016(5)$ & $-0.0005(5)$ \\
\hline $\mathrm{C} 24$ & $0.0215(7)$ & $0.0210(7)$ & $0.0257(7)$ & $0.0010(5)$ & $0.0004(6)$ & $0.0025(6)$ \\
\hline $\mathrm{C} 25$ & $0.0195(6)$ & $0.0208(7)$ & $0.0228(7)$ & 0.0024 & $0.0014(5)$ & $-0.0022(5)$ \\
\hline N22 & $0.0226(6)$ & $0.0262(7)$ & $0.0205(6)$ & $-0.0024(5)$ & $0.0029(5)$ & $0.0018(5)$ \\
\hline $\mathrm{C} 26$ & $0.0216(7)$ & $0.0333(8)$ & $0.0195(7)$ & $-0.0043(6)$ & $0.0037(5)$ & $-0.0005(6)$ \\
\hline $\mathrm{C} 27$ & $0.0308(8)$ & $0.0293(8)$ & $0.0269(7)$ & $-0.0040(6)$ & $0.0021(6)$ & $0.0076(6)$ \\
\hline
\end{tabular}

Geometric parameters $(\AA, \stackrel{\circ}{)})$

\begin{tabular}{llll}
\hline Co1-N1 & $1.9521(13)$ & $\mathrm{C} 16-\mathrm{H} 16 \mathrm{C}$ & 0.9800 \\
Co1-N2 & $1.9535(14)$ & $\mathrm{C} 17-\mathrm{H} 17 \mathrm{~A}$ & 0.9800 \\
$\mathrm{Co1}-\mathrm{N} 11$ & $2.0057(12)$ & $\mathrm{C} 17-\mathrm{H} 17 \mathrm{~B}$ & 0.9800 \\
$\mathrm{Co1}-\mathrm{N} 21$ & $\mathrm{C} 17-\mathrm{H} 17 \mathrm{C}$ & 0.9800 \\
$\mathrm{~N} 1-\mathrm{C} 1$ & $2.0013(12)$ & $\mathrm{N} 21-\mathrm{C} 21$ & $1.3579(18)$ \\
$\mathrm{C} 1-\mathrm{S} 1$ & $1.170(2)$ & $\mathrm{N} 21-\mathrm{C} 25$ & $1.3522(19)$ \\
$\mathrm{N} 2-\mathrm{C} 2$ & $1.6164(15)$ & $\mathrm{C} 21-\mathrm{H} 21$ & 0.9500 \\
$\mathrm{C} 2-\mathrm{S} 2$ & $1.166(2)$ & $\mathrm{C} 21-\mathrm{C} 22$ & $1.371(2)$ \\
$\mathrm{N} 11-\mathrm{C} 11$ & $1.6253(16)$ & $\mathrm{C} 22-\mathrm{H} 22$ & 0.9500 \\
$\mathrm{~N} 11-\mathrm{C} 15$ & $1.3502(18)$ & $\mathrm{C} 22-\mathrm{C} 23$ & $1.415(2)$
\end{tabular}




\begin{tabular}{|c|c|c|c|}
\hline $\mathrm{C} 11-\mathrm{H} 11$ & 0.9500 & $\mathrm{C} 23-\mathrm{C} 24$ & $1.420(2)$ \\
\hline $\mathrm{C} 11-\mathrm{C} 12$ & $1.372(2)$ & $\mathrm{C} 23-\mathrm{N} 22$ & $1.3472(18)$ \\
\hline $\mathrm{C} 12-\mathrm{H} 12$ & 0.9500 & $\mathrm{C} 24-\mathrm{H} 24$ & 0.9500 \\
\hline $\mathrm{C} 12-\mathrm{C} 13$ & $1.413(2)$ & $\mathrm{C} 24-\mathrm{C} 25$ & $1.370(2)$ \\
\hline $\mathrm{C} 13-\mathrm{C} 14$ & $1.418(2)$ & $\mathrm{C} 25-\mathrm{H} 25$ & 0.9500 \\
\hline $\mathrm{C} 13-\mathrm{N} 12$ & $1.3498(19)$ & $\mathrm{N} 22-\mathrm{C} 26$ & $1.4590(19)$ \\
\hline $\mathrm{C} 14-\mathrm{H} 14$ & 0.9500 & $\mathrm{~N} 22-\mathrm{C} 27$ & $1.461(2)$ \\
\hline $\mathrm{C} 14-\mathrm{C} 15$ & $1.371(2)$ & $\mathrm{C} 26-\mathrm{H} 26 \mathrm{~A}$ & 0.9800 \\
\hline $\mathrm{C} 15-\mathrm{H} 15$ & 0.9500 & $\mathrm{C} 26-\mathrm{H} 26 \mathrm{~B}$ & 0.9800 \\
\hline $\mathrm{N} 12-\mathrm{C} 16$ & $1.459(2)$ & $\mathrm{C} 26-\mathrm{H} 26 \mathrm{C}$ & 0.9800 \\
\hline $\mathrm{N} 12-\mathrm{C} 17$ & $1.457(2)$ & $\mathrm{C} 27-\mathrm{H} 27 \mathrm{~A}$ & 0.9800 \\
\hline $\mathrm{C} 16-\mathrm{H} 16 \mathrm{~A}$ & 0.9800 & $\mathrm{C} 27-\mathrm{H} 27 \mathrm{~B}$ & 0.9800 \\
\hline $\mathrm{C} 16-\mathrm{H} 16 \mathrm{~B}$ & 0.9800 & $\mathrm{C} 27-\mathrm{H} 27 \mathrm{C}$ & 0.9800 \\
\hline $\mathrm{N} 1-\mathrm{Co} 1-\mathrm{N} 2$ & $117.81(6)$ & $\mathrm{N} 12-\mathrm{C} 17-\mathrm{H} 17 \mathrm{~B}$ & 109.5 \\
\hline $\mathrm{N} 1-\mathrm{Co} 1-\mathrm{N} 11$ & $105.83(5)$ & $\mathrm{N} 12-\mathrm{C} 17-\mathrm{H} 17 \mathrm{C}$ & 109.5 \\
\hline $\mathrm{N} 1-\mathrm{Co} 1-\mathrm{N} 21$ & $105.41(5)$ & $\mathrm{H} 17 \mathrm{~A}-\mathrm{C} 17-\mathrm{H} 17 \mathrm{~B}$ & 109.5 \\
\hline $\mathrm{N} 2-\mathrm{Co} 1-\mathrm{N} 11$ & $108.82(5)$ & $\mathrm{H} 17 \mathrm{~A}-\mathrm{C} 17-\mathrm{H} 17 \mathrm{C}$ & 109.5 \\
\hline $\mathrm{N} 2-\mathrm{Co} 1-\mathrm{N} 21$ & $106.71(5)$ & $\mathrm{H} 17 \mathrm{~B}-\mathrm{C} 17-\mathrm{H} 17 \mathrm{C}$ & 109.5 \\
\hline $\mathrm{N} 21-\mathrm{Co} 1-\mathrm{N} 11$ & $112.36(5)$ & $\mathrm{C} 21-\mathrm{N} 21-\mathrm{Co} 1$ & $117.41(10)$ \\
\hline $\mathrm{C} 1-\mathrm{N} 1-\mathrm{Co} 1$ & $168.56(12)$ & $\mathrm{C} 25-\mathrm{N} 21-\mathrm{Co} 1$ & $124.79(10)$ \\
\hline $\mathrm{N} 1-\mathrm{C} 1-\mathrm{S} 1$ & $178.72(14)$ & $\mathrm{C} 25-\mathrm{N} 21-\mathrm{C} 21$ & $116.22(12)$ \\
\hline $\mathrm{C} 2-\mathrm{N} 2-\mathrm{Co} 1$ & $175.34(13)$ & $\mathrm{N} 21-\mathrm{C} 21-\mathrm{H} 21$ & 118.0 \\
\hline $\mathrm{N} 2-\mathrm{C} 2-\mathrm{S} 2$ & $178.29(15)$ & $\mathrm{N} 21-\mathrm{C} 21-\mathrm{C} 22$ & 124.03 \\
\hline $\mathrm{C} 11-\mathrm{N} 11-\mathrm{Co} 1$ & $119.59(10)$ & $\mathrm{C} 22-\mathrm{C} 21-\mathrm{H} 21$ & 118.0 \\
\hline $\mathrm{C} 15-\mathrm{N} 11-\mathrm{Co} 1$ & $123.89(10)$ & $\mathrm{C} 21-\mathrm{C} 22-\mathrm{H} 22$ & 120.1 \\
\hline $\mathrm{C} 15-\mathrm{N} 11-\mathrm{C} 11$ & $116.49(12)$ & $\mathrm{C} 21-\mathrm{C} 22-\mathrm{C} 23$ & $119.87(13)$ \\
\hline $\mathrm{N} 11-\mathrm{C} 11-\mathrm{H} 11$ & 118.1 & $\mathrm{C} 23-\mathrm{C} 22-\mathrm{H} 22$ & 120.1 \\
\hline $\mathrm{N} 11-\mathrm{C} 11-\mathrm{C} 12$ & $123.84(13)$ & $\mathrm{C} 22-\mathrm{C} 23-\mathrm{C} 24$ & $115.75(13)$ \\
\hline $\mathrm{C} 12-\mathrm{C} 11-\mathrm{H} 11$ & 118.1 & $\mathrm{~N} 22-\mathrm{C} 23-\mathrm{C} 22$ & $122.41(13)$ \\
\hline $\mathrm{C} 11-\mathrm{C} 12-\mathrm{H} 12$ & 120.1 & $\mathrm{~N} 22-\mathrm{C} 23-\mathrm{C} 24$ & $121.80(14)$ \\
\hline $\mathrm{C} 11-\mathrm{C} 12-\mathrm{C} 13$ & $119.85(14)$ & $\mathrm{C} 23-\mathrm{C} 24-\mathrm{H} 24$ & 120.0 \\
\hline $\mathrm{C} 13-\mathrm{C} 12-\mathrm{H} 12$ & 120.1 & $\mathrm{C} 25-\mathrm{C} 24-\mathrm{C} 23$ & $120.00(14)$ \\
\hline $\mathrm{C} 12-\mathrm{C} 13-\mathrm{C} 14$ & $116.08(13)$ & $\mathrm{C} 25-\mathrm{C} 24-\mathrm{H} 24$ & 120.0 \\
\hline $\mathrm{N} 12-\mathrm{C} 13-\mathrm{C} 12$ & $121.80(14)$ & $\mathrm{N} 21-\mathrm{C} 25-\mathrm{C} 24$ & $123.92(13)$ \\
\hline $\mathrm{N} 12-\mathrm{C} 13-\mathrm{C} 14$ & $122.12(14)$ & $\mathrm{N} 21-\mathrm{C} 25-\mathrm{H} 25$ & 118.0 \\
\hline $\mathrm{C} 13-\mathrm{C} 14-\mathrm{H} 14$ & 120.2 & $\mathrm{C} 24-\mathrm{C} 25-\mathrm{H} 25$ & 118.0 \\
\hline $\mathrm{C} 15-\mathrm{C} 14-\mathrm{C} 13$ & $119.54(13)$ & $\mathrm{C} 23-\mathrm{N} 22-\mathrm{C} 26$ & $120.87(13)$ \\
\hline $\mathrm{C} 15-\mathrm{C} 14-\mathrm{H} 14$ & 120.2 & $\mathrm{C} 23-\mathrm{N} 22-\mathrm{C} 27$ & $121.07(13)$ \\
\hline $\mathrm{N} 11-\mathrm{C} 15-\mathrm{C} 14$ & $124.11(13)$ & $\mathrm{C} 26-\mathrm{N} 22-\mathrm{C} 27$ & $118.06(12)$ \\
\hline $\mathrm{N} 11-\mathrm{C} 15-\mathrm{H} 15$ & 117.9 & $\mathrm{~N} 22-\mathrm{C} 26-\mathrm{H} 26 \mathrm{~A}$ & 109.5 \\
\hline $\mathrm{C} 14-\mathrm{C} 15-\mathrm{H} 15$ & 117.9 & $\mathrm{~N} 22-\mathrm{C} 26-\mathrm{H} 26 \mathrm{~B}$ & 109.5 \\
\hline $\mathrm{C} 13-\mathrm{N} 12-\mathrm{C} 16$ & $120.26(13)$ & $\mathrm{N} 22-\mathrm{C} 26-\mathrm{H} 26 \mathrm{C}$ & 109.5 \\
\hline $\mathrm{C} 13-\mathrm{N} 12-\mathrm{C} 17$ & $120.40(13)$ & $\mathrm{H} 26 \mathrm{~A}-\mathrm{C} 26-\mathrm{H} 26 \mathrm{~B}$ & 109.5 \\
\hline $\mathrm{C} 17-\mathrm{N} 12-\mathrm{C} 16$ & $118.32(13)$ & $\mathrm{H} 26 \mathrm{~A}-\mathrm{C} 26-\mathrm{H} 26 \mathrm{C}$ & 109.5 \\
\hline $\mathrm{N} 12-\mathrm{C} 16-\mathrm{H} 16 \mathrm{~A}$ & 109.5 & $\mathrm{H} 26 \mathrm{~B}-\mathrm{C} 26-\mathrm{H} 26 \mathrm{C}$ & 109.5 \\
\hline $\mathrm{N} 12-\mathrm{C} 16-\mathrm{H} 16 \mathrm{~B}$ & 109.5 & $\mathrm{~N} 22-\mathrm{C} 27-\mathrm{H} 27 \mathrm{~A}$ & 109.5 \\
\hline $\mathrm{N} 12-\mathrm{C} 16-\mathrm{H} 16 \mathrm{C}$ & 109.5 & $\mathrm{~N} 22-\mathrm{C} 27-\mathrm{H} 27 \mathrm{~B}$ & 109.5 \\
\hline
\end{tabular}


supporting information

\begin{tabular}{llll} 
H16A-C16-H16B & 109.5 & N22-C27-H27C & 109.5 \\
H16A-C16-H16C & 109.5 & H27A-C27-H27B & 109.5 \\
H16B-C16-H16C & 109.5 & H27A-C27-H27C & 109.5 \\
N12-C17-H17A & 109.5 & H27B-C27-H27C & 109.5 \\
\hline
\end{tabular}

Hydrogen-bond geometry $\left(A,{ }^{\circ}\right)$

\begin{tabular}{lllll}
\hline$D-\mathrm{H} \cdots A$ & $D-\mathrm{H}$ & $\mathrm{H} \cdots A$ & $D \cdots A$ & $D-\mathrm{H} \cdots A$ \\
\hline $\mathrm{C} 11-\mathrm{H} 11 \cdots \mathrm{S} 1^{\mathrm{i}}$ & 0.95 & 2.87 & $3.7312(15)$ & 152 \\
$\mathrm{C} 16-\mathrm{H} 16 C \cdots \mathrm{S} 2^{\mathrm{ii}}$ & 0.98 & 3.01 & $3.9663(16)$ & 166 \\
$\mathrm{C} 21-\mathrm{H} 21 \cdots \mathrm{S} 1^{\mathrm{iii}}$ & 0.95 & 2.92 & $3.7888(15)$ & 153 \\
$\mathrm{C} 22-\mathrm{H} 22 \cdots \mathrm{N} 1^{\text {iv }}$ & 0.95 & 2.64 & $3.5448(19)$ & 159 \\
$\mathrm{C} 26-\mathrm{H} 26 A \cdots \mathrm{N} 2^{\mathrm{iv}}$ & 0.98 & 2.70 & $3.531(2)$ & 143 \\
$\mathrm{C} 27-\mathrm{H} 27 B \cdots \mathrm{S} 2^{\text {iv }}$ & 0.98 & 2.98 & $3.7568(17)$ & 137
\end{tabular}

Symmetry codes: (i) $-x+2,-y+1,-z+2$; (ii) $-x+1,-y+1,-z+2$; (iii) $-x+2, y-1 / 2,-z+3 / 2$; (iv) $x,-y+1 / 2, z-1 / 2$. 